\title{
水氧化释放质子与 $\mathrm{H}^{+}-\mathrm{ATP}$ 酶复合 体的 $\mathrm{CF}_{\text {。 }}$ 之间的联系*
}

\author{
吴少龙 马正平 李有则 \\ (中国科学院上海植物生理研究所, 上海 200032)
}

关调质子转移、毫秒延迟发光、膜上区域化质子

光合电子传递链有两个释放质子的部位, 一是位于类衰体膜内侧的光系统 II (Photosystem II, PSII) 放氧复合体氧化水时释放质子 $\left(\mathrm{H}_{\mathrm{H}_{2} \mathrm{O}}^{+}\right)$, 另一是与光系统 I(Photosystem I, PSI) 相 联系的质醌 (Plastoquinone, $\mathrm{PQ}$ ) 氧化还原跨膜携带质子在类亳体膜内侧释放 $\left(\mathrm{H}_{\mathrm{PQ}}^{+} \mathrm{H}_{2}\right)$. 这

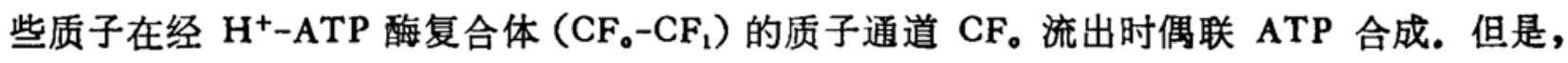
这些质子如何从它们的释放部位传导到 $\mathrm{CF}_{\text {。 }}$ (是经过类衰体腔内水相还是在膜上有专门的途 径)至今仍是一个不清楚而争论激烈的问题 ${ }^{[1]}$. 尤其是分布于垛叠区的 PSII 放氧复合体所释

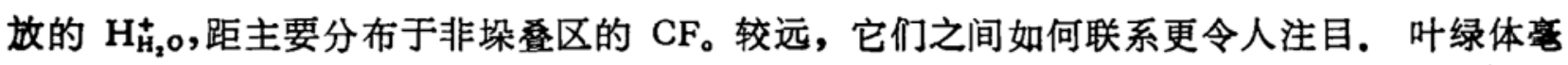
秒延迟发光 (ms Delayed light emission, ms DLE) 是光反应中心电荷分离后再重组时所发 出的苂光, 主要来自 $\mathrm{PSII}^{[2]}$, 与电子传递和质子传导有密切的关系. 本文从主要作用于 $\mathrm{CF}_{\text {。 }}$ 的能量传递抑制剂氯化三苯锡 (Triphenyltin chloride, TPT) ${ }^{[3]}$ 对叶绿体 ms DLE 的促进 作用人手, 从一新的角度探讨 $\mathrm{H}_{\mathrm{H}_{2}}^{+}$。 与 $\mathrm{CF}_{0}$ 的联系.

\section{一、材料与方法}

菠菜 (Spinacia oleracia L.) 叶绿体和去除 $\mathrm{CF}_{1}$ 的类震体残缺膜制备、叶绿素含量测 定、质子外流速度及跨膜 $\Delta \mathrm{pH}$ 测定、光合磷酸化活力测定等均同文献 $[3,4]$. PS Il 颗粒制备 按照 BBY 方法略作修改(第一次与 Triton X-100 保温时间从 $30 \mathrm{~min}$ 减为 $10 \mathrm{~min}$ ).

\section{二、结果与讨论}

1. TPT 对叶绿体 ms DLE 和质子传导的影响 TPT 和二环已基碳二亚胺 (Dicyclohexylcarbodiimide, DCCD) 均是主要作用于 $\mathrm{CF}_{0}$ 的能量传递抑制剂 ${ }^{[3]}$, 但低浓度的 TPT 明显促进叶绿体的 ms DLE, 而 DCCD 无此作用(图 1A). TPT 对叶绿体的基础或解联电

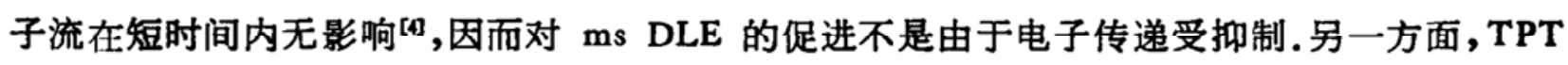
和 DCCD 均加速正常叶绿体类嚲体腔内质子 $\left(\mathrm{H}_{\mathrm{ia}}^{+}\right.$) 的外流(图 $1 \mathrm{~B}$ ). . 虽然 DCCD 的作用 浓度要比 TPT 大得多, 但在明显加速 $\mathrm{H}_{\mathrm{i}}^{+}$外流的浓度 $(25 \mu \mathrm{mol} / \mathrm{L})$ 下并不表现对 $\mathrm{ms}$ DLE 的促进(图 1A), 因而 TPT 对 ms DLE 的促进不能认为是加速 $\mathrm{H}_{\mathrm{i}}^{+}$外流所致. 再一方面, TPT 和 DCCD 又都可堵塞类毫体残缺膜因 $\mathrm{CF}_{1}$ 去除而引起的质子泄漏, 败复跨㬺 $\Delta \mathrm{pH}$ 的

本文 1990 年 2 月 20 日收到. 1990.年 7 月 1 日收到焦改椈.

- 国家自然科学基金炵助项目. 

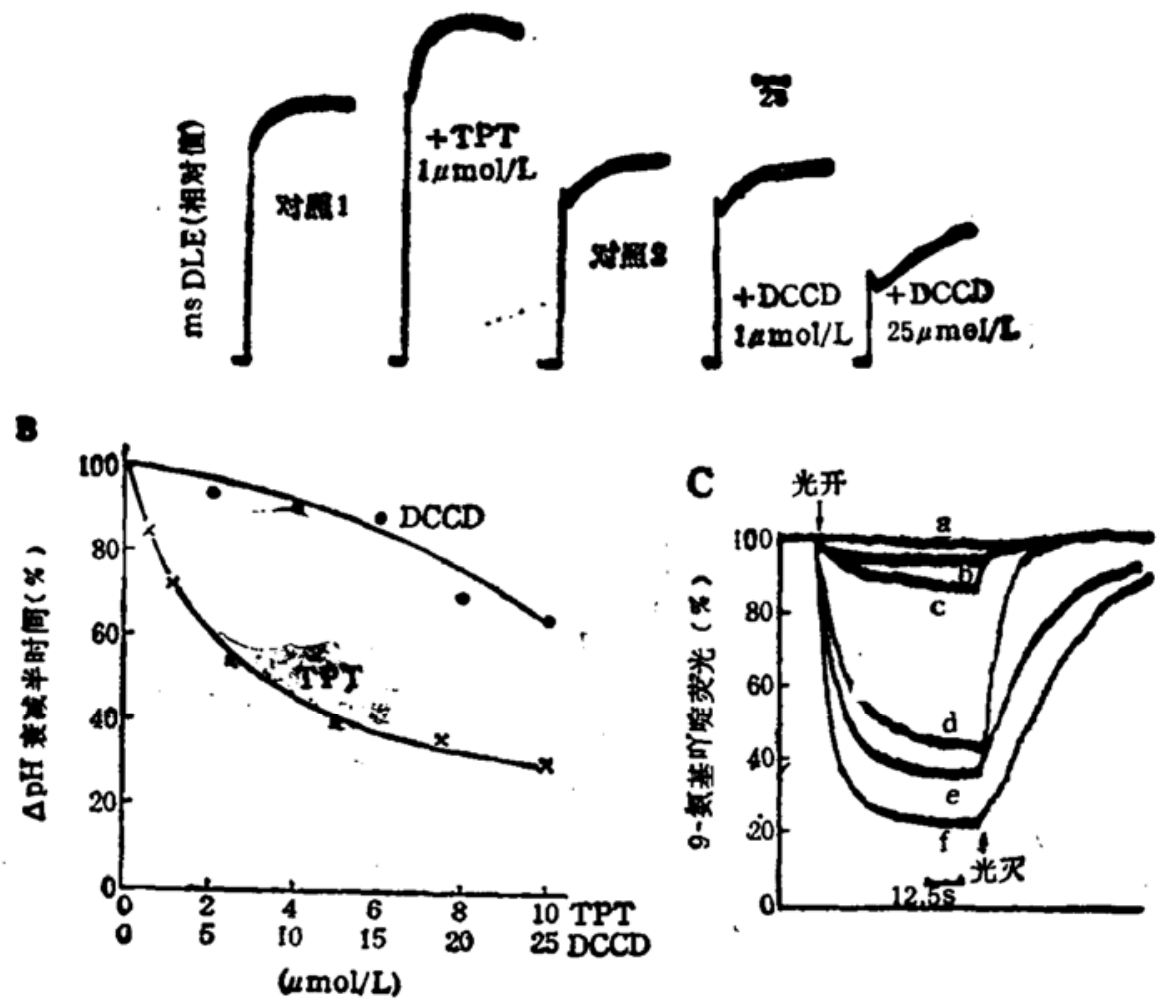

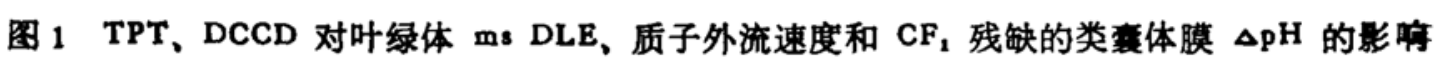

A. ms DLE, 反应液含 $50 \mathrm{mmol} / \mathrm{LT}$ ricine $(\mathrm{pH} 8.0) 、 20 \mathrm{mmol} / \mathrm{L}$ 鹿糖、5 mmol/L $\mathrm{MgCl}_{2} 、 10 \mathrm{mmol} /$ L KCl、0.1 mmol/L 甲基策晶 (Methyl Viologen, MV)、30 $\mu \mathrm{g} / \mathrm{ml}$ 叶绿系的叶绿体; B. 叶绿体质

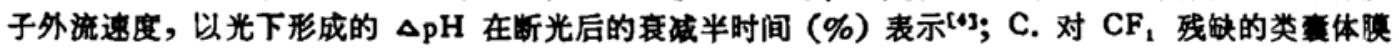

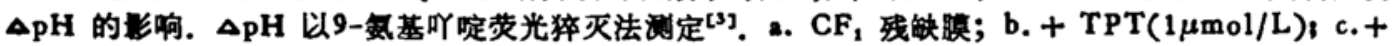
$D C C D(5 \mu \mathrm{mol} / L) ;$ d. $+\operatorname{TPT}(5 \mu \mathrm{mol} / \mathrm{L}) ;$ e. $+\operatorname{DCCD}(50 \mu \mathrm{mol} / \mathrm{L}) ;$ f. 对照叶绿体. B. C 反应液同

A. 另外含 7.5 $\mu \mathrm{mol} / \mathrm{L} 9$-氨基叫啶. 对盟 2 和所有 $\mathrm{DCCD}$ 处理都经过 $20^{\circ} \mathrm{C} 20 \mathrm{~min}$ 保温

形成(图 1C), 然而 DCCD 并没有促进 ms DLE 的作用, 因而 TPT 对 ms DLE 的促进又 不能简单地归结为是由于质子跨膜向外传导受阻, 况且在正常叶绿体 TPT 是加速质子向外 传导的(图 1B).

2. 解联状态下 TPT 对 ms DLE 的影䛲考虑到 ms DLE 主要来自 PS II 反应中 心的电荷重组, 则 TPT 对 ms DLE 的促进是否是由于与 PSII 放氧复合体有直接的相互作 用? 为此我们分离了具有较高放氧活性的 PSII 颗粒(所含 $\mathrm{CF}_{0}-\mathrm{CF}_{1}$ 复合体极少). TPT 对 这颗柆的 ms DLE 没有明显的作用 (图 2A), 表明 TPT 与 PSII 放氧复合体无直接的相互 作用: TPT 是作用于 $\mathrm{CF}_{\text {。 }}$ 之后才引起 $\mathrm{ms}$ DLE 的增加, 因而在 PSII 放氧放合体与 $\mathrm{CF}_{0}$ 之 间可能存在某种联系. 当在尼日利亚菌素、 $\mathrm{NH}_{4} \mathrm{Cl}$ 或短杆菌肽 $\mathrm{D}$ 的解联作用下, TPT 对叶绿 体的 ms DLE 的促进作用消失(图 2B), 这说明这种联系与质子传导有关.

3. 高盐浓度或低 pH 介质中 TPT 对 ms DLE 的狸响 在图 2B 所用的解联剂浓度 下已完全解联, 跨膜质子或膜上区域化质子均被消去, 不能区分 PSII 放氧复合体与 CF。之 间的质子联系的性质. Dilley 等的试验表明在高盐或低介质中, 膜上的区域化质子可以被消 去 ${ }^{[6]}$. 我们也发现只有在碱性 $\mathrm{pH}$ 介质下才能测到膜上区域化质子的存在, 而在酸性 $\mathrm{pH}$ 介 质下则否; 高盐介质下膜上区域化质子也趋于消失(未发表的结果)。图 3 表明只有在偏碱性 低盐介质下 TPT 才促进叶绿体的 ms DLE, 而在低 $\mathrm{pH}$ 介质或高盐介质下 TPT 对 ms DLE 
的促进作用不再出现,这说明上述所提及的质子联系与膜上区域化质子有关.

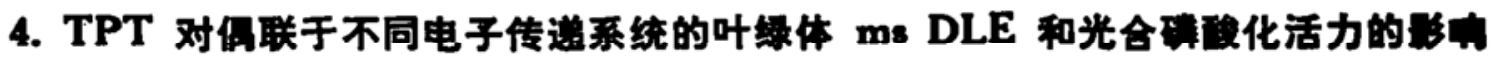

虽然由于叶绿体的 ms DLE 主要来自 PSII, 可以设想上述的 PSII 放氧复合体与 $\mathrm{CF}_{\text {。之 }}$ 间的质子联系主要涉及 $\mathrm{H}_{\mathrm{H}_{2} \mathrm{O}}^{+}$，但还需要进 一步的说明. 利用不同的人工电子供受体可 以使叶绿体进行不同系统的电子传递. 图 4 表明 TPT 对涉及 PSII 电子传递的叶绿体 m8 DLE 仍有促进, 而对与 PSI 电子传递相 偶联的叶绿体 ms DLE 则不促进, 这说明 TPT 促进 ms DLE 与 $\mathrm{H}_{\mathrm{H}_{2}}^{+} \mathrm{O}$ 的关系更为密 切. 从 TPT 对偶联 PSII 电子传递的叶绿体 光合磷酸化的抑制比对偶联于 PS I 或 PS I + PSII 的更为严重和敏感也说明 $\mathrm{H}_{\mathrm{H}_{2}}^{+} \mathrm{O}$ 与 CF。之问有更直接的联系(图 5).

总结以上的结果, 可以认为 $\mathrm{H}_{\mathrm{H}_{2}}^{+} \mathrm{O}$ 与 CF。之间可以通过膜上的区域化质子相联

系, $\mathrm{H}_{\mathrm{H}_{2}}^{+} \mathrm{O}$ 可经由膜上的专门途径 (即膜上区域化质子传导途径) 直接从释放位点传导到 CF. 而用于 ATP 的合成. TPT 作用于 CF。后, 可能是阻断了这条质子传导途径, 从而表现出 ms DLE 的增强.

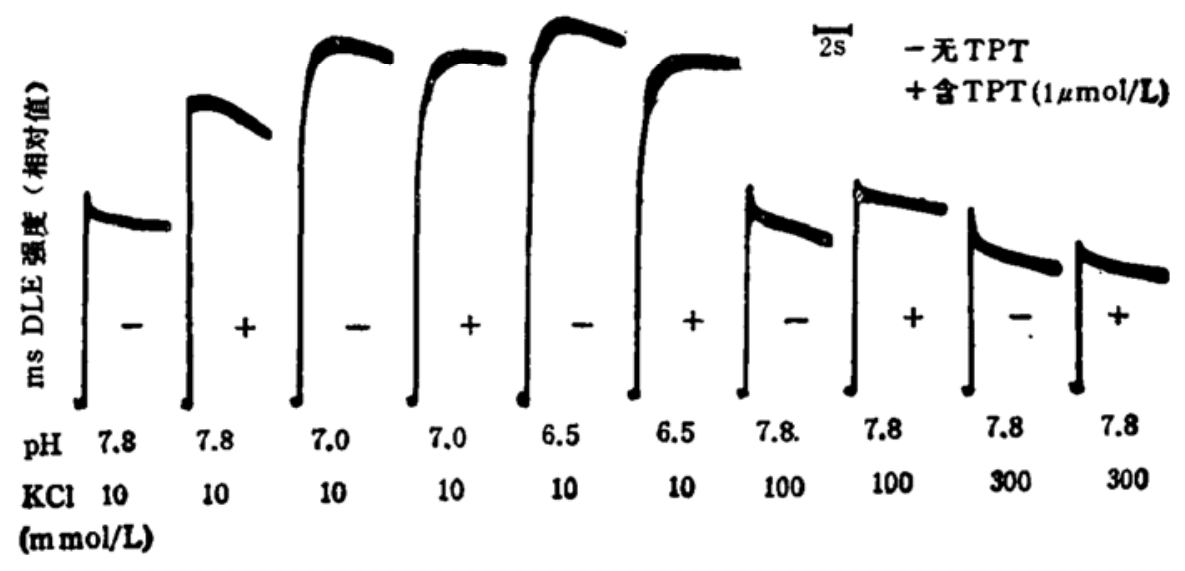

图 3 在不同介质 $\mathrm{pH}$ 和盐浓度条件下 TPT 对叶绿体 $\mathrm{M}$ DLE 的影响

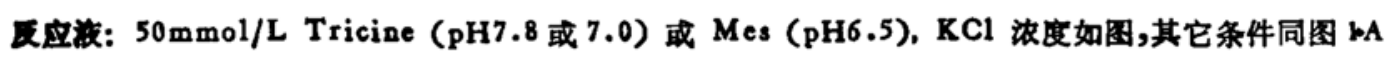

关于膜上区域化质子的研究近年来也有不少的进展, 其中较突出的是 Dilley 实验室的工 作 ${ }^{[6,7]}$, 他们主要应用醋酸䣲修饰的方法, 认为 $\mathrm{H}_{\mathrm{H}_{2}}^{+} \mathrm{O}$ 和 $\mathrm{H}_{\mathrm{PQH}_{2}}^{+}$都可通过膜上区域化质子传导 途径与 CF. 发生直接的联系. 我们从作用于 CF。的 TPT 对叶绿体 ms DLE 的影响来研 究 $\mathrm{H}_{\mathrm{H}_{2}}^{+} \mathrm{O}$ 与 $\mathrm{CF}_{0}$ 的联系是一种新的尝试, 从一个新的角度表明 $\mathrm{H}_{\mathrm{H}_{2}}^{+} \mathrm{O}$ 与 $\mathrm{CF}_{0}$ 之间可以有直 接的联系. 至于 $\mathrm{H}_{\mathrm{H}_{2}}^{+} \mathrm{O}$ 与 $\mathrm{CF}$ 。之间膜上区域化质子传导途径的结构基础, 目前了解得很少, 需要进一步深人研究. 


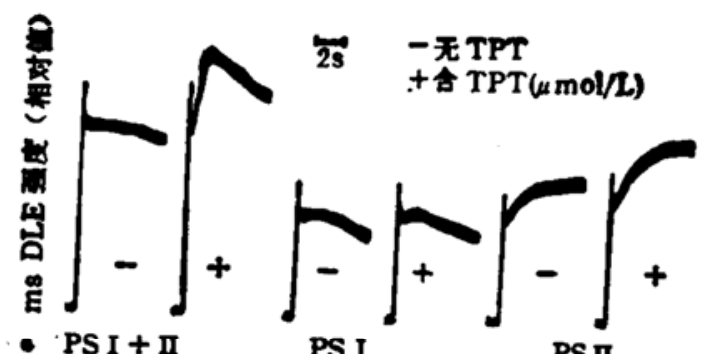

图4 TPT 对偶联于不同电子传递系统的叶绿体 ms DLE 的影响

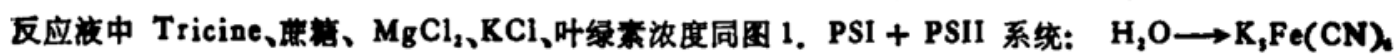
$(1 \mathrm{mmol} / \mathrm{L})$; PSI 系统: 还原奆 2, 6-dichlorophenol indophenol(DPIP) $\rightarrow$ MV, 反应液含 0.2 mmol/L DPIP、1.33mmol/L 还原态抗坏血酸、0.1 mmol/L MV、 $5 \mu \mathrm{mol} / \mathrm{L}$ Diuron; PSIl 系绕: $\mathrm{H}_{2} \mathrm{O} \longrightarrow$ 年化态 $p$-phenylene diamine $(\mathrm{PD})$, 反应液含 $0.1 \mathrm{mmol} / \mathrm{L} \mathrm{PD} 、 1 \mathrm{mmol} / \mathrm{L} \mathrm{K}, \mathrm{Fe}(\mathrm{CN})$ 。 $5 \mu \mathrm{mol} / \mathrm{L}$ dibromothv-soquinone

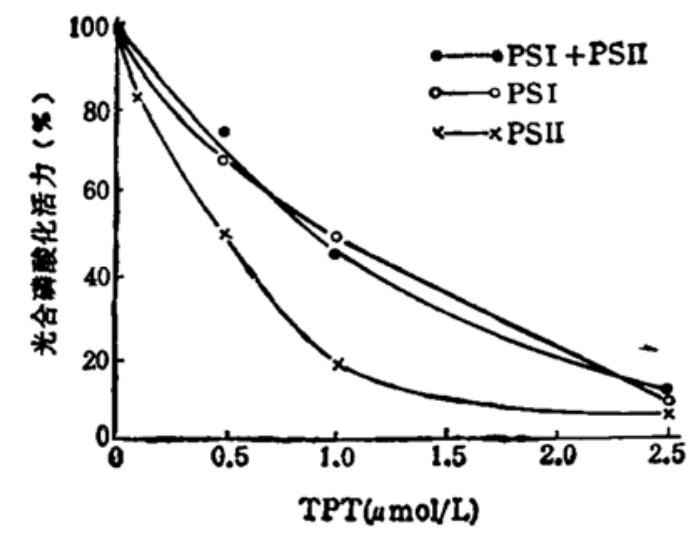

图5TPT 对偶联于不同电子传递系统的叶绿体光合磷酸化活力的影响

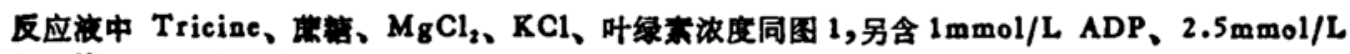
$\mathrm{Pi}^{32} \mathrm{Pi}$ 约 $10^{6}$ 脉冲数/min. PSI + PSII 系统: $\mathrm{H}_{2} \mathrm{O} \longrightarrow \mathrm{MV}(0.1 \mathrm{mmol} / \mathrm{L})$, PSI 和 PSII 系统 同图 4

\section{告考文献}

[1] Ferguson, S. J., Biochim. Biophy. Acta, 811(1985), 47-95.

\{2] Jursinic, P. A., in Light Emission by Plant and Bacteria (Eds. Govindjee et al.). Academic Prese, Orlando, 1986, 291-328.

[3] 吴少龙典,植物生理学报,15(1989),76-82.

[4] 吴少龙、本有则,植物生理学报, 15(1989), 125-131.

[5] Berthod, D. A. et al., FEBS Lett., 134(1981), 231-234.

[6] Dilley, R. A. et al., Ann. Rev. Plant Physiol., 38(1987), 347-389.

[7] Allautt, F. C. T. et al., Photosyn. Res., 20(1989), 161-172. 